PROCEEDINGS OF THE

AMERICAN MATHEMATICAL SOCIETY

Volume 127, Number 2, February 1999, Pages 473-479

S 0002-9939(99)04829-7

\title{
THE SADDLE POINT PROPERTY FOR FOCUSING SELFSIMILAR SOLUTIONS IN A FREE BOUNDARY PROBLEM
}

\author{
CLAUDE-MICHEL BRAUNER, JOSEPHUS HULSHOF, \\ AND CLAUDINE SCHMIDT-LAINÉ \\ (Communicated by Jeffrey Rauch)
}

\begin{abstract}
We establish the saddle point property of the focusing selfsimilar solution of a free boundary problem for the heat equation with free boundary conditions given by $u=0$ and $\frac{\partial u}{\partial \nu}=1$.
\end{abstract}

\section{INTRODUCTION}

In this note we consider a one-phase free boundary problem (FBP) for the heat equation

$$
u_{t}=\Delta u, \quad u=u(x, t), \quad x=\left(x_{1}, \ldots, x_{N}\right) \in \Omega_{t}, \quad t>0,
$$

where

$$
\Delta=\frac{\partial^{2}}{\partial x_{1}^{2}}+\cdots+\frac{\partial^{2}}{\partial x_{N}^{2}},
$$

and $\Omega_{t}$ is a domain in $\mathbb{R}^{N}$, with (free) boundary $\Gamma_{t}$. A typical free boundary condition one encounters in combustion models [12] is that at the free boundary both $u$ and the outward normal derivative $\frac{\partial u}{\partial \nu}$ are prescribed constants, e.g.

$$
u=q>0 \quad \text { and } \quad \frac{\partial u}{\partial \nu}=1 \quad \text { on } \quad \Gamma_{t} .
$$

Wellposedness of this free boundary problem and related problems is studied in $[2,3,9,10]$. In the radial case uniqueness results are established in [9].

Problem (1.1)(1.2) admits both travelling fronts as well as selfsimilar focusing. In one dimension the travelling wave solution is given by

$$
u(x, t)=q \exp \left(\frac{q x+t}{q^{2}}\right), \quad \Omega_{t}=\{q x+t<0\}, \quad \Gamma_{t}=\{q x+t=0\} .
$$

This solution is invariant under translations and may be seen as an equilibrium in the travelling wave variables $\xi=x+c t, \tau=t$, with speed $c=1 / q$. The stability properties of such travelling waves have been studied from a global point of view

Received by the editors May 21, 1997.

1991 Mathematics Subject Classification. Primary 35K55, 35K65, 80A25.

Key words and phrases. Free boundary problem, similarity variables, linearization, analytic semigroups, fully nonlinear equations, saddle point property.

We are grateful for the support of the Universities of Leiden and Bordeaux I and of the HCMproject "Nonlinear PDE-'s" (ERBCHRXCT 940-618). The visit of the first and the third author to Leiden was supported by the ESF/FBP program. 
in the one-dimensional case in [10]. In [5] two of the authors have developed with A. Lunardi a new method based on the elimination of the front and the reformulation of the problem as a fully nonlinear equation in a suitable Banach space. The method turns out to be quite efficient for multidimensional stability problems $[6,7,8]$.

If one considers scalings instead of translations, one easily finds the radially symmetric selfsimilar solution

$$
u(x, t)=q+\sqrt{T-t} f(\eta), \eta=\frac{|x|}{\sqrt{T-t}},
$$

with $f(\eta)$ satisfying

$$
\begin{gathered}
f^{\prime \prime}(\eta)+\frac{N-1}{\eta} f^{\prime}(\eta)+\frac{1}{2} f=\frac{1}{2} \eta f^{\prime} \text { for } 0 \leq \eta \leq b ; \\
f^{\prime}(0)=f(b)=0, f^{\prime}(b)=1 .
\end{gathered}
$$

This uniquely determines the value of $b>0$ for which exactly one solution $f$ of $(1.5)(1.6)$ with $f<0$ on $[0, b)$ exists, and yields a similarity solution of (1.1)(1.2) which "focuses" in the origin at $t=T$, the domain being given by

$$
\Omega_{t}=\left\{|x|^{2}<b^{2}(T-t)\right\} .
$$

For this focusing solution a global stability analysis has been done in the radial case, see [11] for $N=1$ and [9] for $N>1$. The result is that if the solution focuses at time $t=T$, then, in the appropriate selfsimilar variables, the solution converges to the selfsimilar solution given by (1.4).

In this note we adapt the methods in $[5,6]$ to linearize the free boundary problem in its selfsimilar variables around the selfsimilar profile $f$ and establish the existence of a stable and unstable manifold. The unstable manifold is not empty, because the free parameter $T$ in (1.4) always leads to $\lambda=1$ being an eigenvalue. This is reminiscent of the travelling wave case, where, due to the translation invariance, $\lambda=0$ is always an eigenvalue. For the radial case we show that all the other eigenvalues are negative. In one dimension, we also consider the nonradial, i.e. the nonsymmetric case. Here we find that $\lambda=\frac{1}{2}$ is also a positive eigenvalue. This is because not only the focusing time, but also the focusing point is now a free parameter. All the other eigenvalues are again negative.

We have restricted the analysis in this paper to radial solutions. This is a stepping stone for a stability analysis in the class of all solutions, radial and nonradial, with clear extension to starshaped domains. This however requires new nontrivial results about invariant manifolds in fully nonlinear parabolic problems [13] and will be the subject of a forthcoming paper [4].

\section{THE LINEARIZATION PROCEDURE IN THE RADIAL CASE}

From here on we assume without loss of generality that $q=0$, and transform the radial focusing problem to selfsimilar variables. Thus, if

$$
\Omega_{t}=\{r=|x|<\xi(t)\}, \quad u(x, t)=u(r, t),
$$

we set

$$
\tilde{r}=\frac{r}{(T-t)^{\frac{1}{2}}}, \quad \tilde{t}=-\log (T-t), \quad \tilde{u}(\tilde{r}, \tilde{t})=\frac{u(r, t)}{(T-t)^{\frac{1}{2}}}, \quad \tilde{\xi}(\tilde{t})=\frac{\xi(t)}{(T-t)^{\frac{1}{2}}} .
$$


Omitting the tildes, we arrive at

$$
u_{t}=u_{r r}+\frac{N-1}{r} u_{r}-\frac{r}{2} u_{r}+\frac{1}{2} u, \quad r<\xi(t)
$$

with (free) boundary conditions

$$
u_{r}(0, t)=u(\xi(t), t)=0, \quad u_{r}(\xi(t), t)=1 .
$$

From [9] we know that Problem $(2.3)(2.4)$ admits a unique stationary negative equilibrium solution, namely the unique negative solution of (1.5)(1.6).

In order to linearize $(2.3)(2.4)$ around this equilibrium, we first make the front steady by setting

$$
z=\frac{b r}{\xi(t)}, \quad \tau=t, \quad \xi(t)=b(1+s(\tau)) .
$$

Note that this change of coordinates is convenient for radial problems: $z$ is a new radial variable which varies from $z=0$ to $z=b$. Next we set

$$
u(r, t)=f(z)+s(\tau) z f^{\prime}(z)+w(z, \tau) .
$$

This splitting is adapted from a similar trick in [5] for travelling waves. Observe that $r=z(1+s)$, so that, in view of $f(r)=f(z(1+s))=f(z)+s z f^{\prime}(z)+O\left(s^{2}\right)$, this splitting is naturally induced by the change of coordinates. Denoting

$$
\Delta_{z} w=w_{z z}+\frac{N-1}{z} w_{z}
$$

this transforms (2.3) into

$$
w_{\tau}(z, \tau)=\mathcal{L} w(z, \tau)+\mathcal{F}_{1}\left(z, \Delta_{z} w(z, \tau), s(\tau)\right)+s^{\prime}(\tau) \mathcal{F}_{2}\left(z, z w_{z}(z, \tau), s(\tau)\right),
$$

where the linear part is given by

$$
\mathcal{L} w=\Delta_{z} w-\frac{z}{2} w_{z}+\frac{1}{2} w
$$

and the nonlinear terms are given by

$$
\begin{gathered}
\mathcal{F}_{1}\left(z, \Delta_{z} w, s\right)=-\frac{s^{2}}{2(1+s)^{2}}\left(z f^{\prime}(z)-f(z)+(s+2) z^{2} f^{\prime \prime}(z)\right) \\
-\frac{s(s+2)}{(1+s)^{2}} \Delta_{z} w, \quad \mathcal{F}_{2}\left(z, z w_{z}, s\right)=\frac{1}{1+s}\left(z w_{z}+s z^{2} f^{\prime \prime}(z)\right) .
\end{gathered}
$$

The free boundary conditions transform into

$$
w_{z}(0, \tau)=0, \quad w(b, \tau)=-b s(\tau), \quad w_{z}(b, \tau)=\left(\frac{b}{2}-\frac{N-1}{b}\right) w(b, \tau) .
$$

From the second condition in (2.10) and (2.7) we derive that, taking $z=b$,

whence

$$
\begin{gathered}
w_{\tau}(b, \tau)=-b s^{\prime}(\tau)=\mathcal{L} w(b, \tau)+\mathcal{F}_{1}\left(b, \Delta_{z} w(b, \tau),-w(b, \tau) / b\right) \\
+s^{\prime}(\tau) \mathcal{F}_{2}\left(b, b w_{z}(b, \tau),-w(b, \tau) / b\right)
\end{gathered}
$$

$$
s^{\prime}(\tau)=-\frac{\mathcal{L} w(b, \tau)+\mathcal{F}_{1}\left(b, \Delta_{z} w(b, \tau),-w(b, \tau) / b\right)}{b+\mathcal{F}_{2}\left(b, w_{z}(b, \tau),-w(b, \tau) / b\right)}
$$


so that (2.7) can be rewritten as the fully nonlinear equation

$$
w_{\tau}(z, \tau)=\mathcal{L} w(z, \tau)+\mathcal{F}\left(z, z w_{z}(z, \tau), \Delta_{z} w(z, \tau), w(b, \tau), b w_{z}(b, \tau), \Delta_{z} w(b, \tau)\right),
$$

with boundary condition

$$
w_{z}(0, \tau)=0, \quad w_{z}(b, \tau)=\left(\frac{b}{2}-\frac{N-1}{b}\right) w(b, \tau) .
$$

Clearly formulas (2.9)-(2.11) define $\mathcal{F}$ as a smooth function of its arguments defined in a neighbourhood of the $z$-axis. Moreover, $\mathcal{F}(z, 0, \ldots, 0)=0$ and on the $z$-axis $\mathcal{F}$ is quadratic with respect to the other variables. Also $\mathcal{F}$ is even in $z$.

\section{FUnCtional ANALYTIC FRAMEWORK}

In this section we transform $(2.12)(2.13)$ in an abstract evolution equation in a suitable Banach space setting [13]. Let $X=C^{0}([0, b])$. The realization $L$ of $\mathcal{L}$ in $X$ with boundary conditions (2.13) has domain

$$
D(L)=\left\{\varphi \in C^{2}([0, b]): \varphi_{z}(0)=0, \varphi_{z}(b)+\left(\frac{N-1}{b}-\frac{b}{2}\right) \varphi(b)=0\right\},
$$

and the operator $L$ is sectorial in $X$. The fact that $L$ is sectorial can be seen as follows: set $z=|x|$ with $x$ in $N$-dimensional Euclidean space (not to be confused with the real variable $x$ we used earlier) and consider $\mathcal{L}$ as the restriction of the differential operator

$$
\tilde{\mathcal{L}} w=\Delta w-\frac{1}{2} x \cdot \nabla w+\frac{1}{2} w
$$

on the ball $B$ with center at the origin and radius $b$, with boundary condition

$$
\tilde{\mathcal{B}} w=\frac{\partial w}{\partial \nu}+\left(\frac{N-1}{b}-\frac{b}{2}\right) w=0 \text { on } \partial B .
$$

The realization $\tilde{L}$ of $\tilde{\mathcal{L}}$ in $\tilde{X}=C^{0}(\bar{B})$ with boundary condition $\tilde{\mathcal{B}} w=0$ has domain

$$
D(L)=\left\{\varphi \in \cap_{p \geq 1} W^{2, p}(B): \tilde{\mathcal{L}} w \in X, \tilde{\mathcal{B}} \varphi=0 \text { on } \partial B\right\},
$$

and is a standard example of a sectorial operator on $\tilde{X}$. Thus its restriction to the closed subspace of radial functions in $\tilde{X}$ is also sectorial, and equivalently so is $L$ on $X$. Alternatively, one may establish directly in the "radial" $z$-variable that $L$ is sectorial. This is done in a slightly different case in [1]. Either way, we have that the domain of $L$ is given by $D(L)$ in (3.3), and the nonlinear part $\mathcal{F}$ defines an analytic map from a closed ball $B_{R}$ in $D(L)$ with sufficiently small radius $R$ centered at the origin. Thus we have that

$$
F: B \subset D(L) \rightarrow X
$$

is a smooth function, and $F$ and its derivative vanish in the origin. Setting $w(t)=$ $w(\cdot, t)$, the problem may be written as

$$
w_{t}=L w+F(w), \text { with initial data } w(0)=w_{0} .
$$

By the theory for fully nonlinear equations (Chapter 8 in [13]) we then have that for every $T>0$ there exists $R>0$ such that (3.4) has a unique solution defined on $[0, T]$ for every $w_{0} \in B_{R}$. To describe the saddle point property of the equilibrium $w=0$ we need the spectrum of $L$. 


\section{THE SADDLE POINT PROPERTY}

The spectral analysis of $L$ leads us to the following eigenvalue problem:

$$
\phi^{\prime \prime}+\left(\frac{N-1}{z}-\frac{z}{2}\right) \phi^{\prime}+\frac{1}{2} \phi=\lambda \phi, \quad \phi^{\prime}(0)=\phi^{\prime}(b)-\left(\frac{b}{2}-\frac{N-1}{b}\right) \phi(b)=0 .
$$

By standard Sturm-Liouville type theory, (4.1) has a sequence

$$
\lambda_{1}>\lambda_{2}>\lambda_{3}>\lambda_{4}>\ldots \downarrow-\infty
$$

of simple eigenvalues which together form the spectrum of $L$. The corresponding eigenfunctions are denoted by

$$
\phi_{1}, \phi_{2}, \phi_{3}, \phi_{4}, \ldots
$$

where $\phi_{n}$ has precisely $n-1$ sign changes. It is easily checked that

$$
\lambda_{1}=1, \quad \phi_{1}(z)=f^{\prime \prime}(z)+\frac{N-1}{z} f^{\prime}(z) .
$$

Now $f$ solves (4.1) with $\lambda=0$, but with the "wrong" boundary condition $f(b)=0$. We claim that consequently $\lambda_{2}<0$. Indeed, every eigenfunction is a multiple of the solution of the ODE in (4.1) with $\phi(0)=1$ and $\phi^{\prime}(0)=0$. In view of the positivity of $-f$ and $f(b)=0$, this solution cannot have a sign change before $z=b$ if $\lambda \geq 0$. Since $\phi_{2}$ must have a sign change, our claim follows.

Let $P$ and $I-P$ be the spectral projections of $L$ with respect to, respectively, $\left\{\lambda_{1}\right\}$ and $\left\{\lambda_{2}, \lambda_{3}, \lambda_{4}, \ldots\right\}$. Then $P(X)$ and $(I-P)(D)$ are the linear unstable manifold and the linear stable manifold of $w=0$. In view of $F$ being smooth on a neighbourhood of $w=0$ in $D(L)$, we may apply Theorem 9.1.4 in [13] to conclude that for every $k \geq 1$ there exist $R>0$ and $C^{k}$-maps

$$
\varphi: B_{R} \cap P(X) \rightarrow(I-P)(D) \text { and } \psi: B_{R} \cap(I-P)(D) \rightarrow P(X),
$$

with $\varphi, \varphi^{\prime}, \psi$ and $\psi^{\prime}$ vanishing in the origin, such that the local unstable and stable manifolds of $w=0$ are given by the graphs of $\varphi$ and $\psi$.

The unstable manifold contains solutions which, in terms of the original variables, do not focus at $t=T$ but at some other time value. The stable manifold contains solutions which converge exponentially to $w=0$. For $u$ and $\xi$ in (2.3) this means that $u(r, t) \rightarrow f(r)$ and $\xi(t) \rightarrow b$, with exponential decay rates. In terms of the original variables (2.1), we have for the free boundary that

$$
\xi(t)=b(T-t)^{\frac{1}{2}}+O(T-t)^{\frac{1}{2}+\mu}
$$

for every $\mu<\lambda_{2}$.

\section{A OnE-Dimensional FBP}

We now consider the case that

$$
\Omega_{t}=\left\{\xi^{-}(t)<x<\xi^{+}(t)\right\},
$$

so that, after

$$
\tilde{x}=\frac{x}{(T-t)^{\frac{1}{2}}}, \quad \tilde{t}=-\log (T-t), \quad \tilde{u}(\tilde{x}, \tilde{t})=\frac{u(x, t)}{(T-t)^{\frac{1}{2}}}, \quad \tilde{\xi}^{ \pm}(\tilde{t})=\frac{\xi^{ \pm}(t)}{(T-t)^{\frac{1}{2}}},
$$


omitting the tildes, we arrive at

$$
u_{t}=u_{x x}-\frac{x}{2} u_{x}+\frac{1}{2} u, \quad \xi^{-}(t)<x<\xi^{+}(t)
$$

with (free) boundary conditions

$$
u\left(\xi^{ \pm}(t), t\right)=0, \quad u_{x}\left(\xi^{ \pm}(t), t\right)= \pm 1
$$

We set

$$
\begin{gathered}
z=\frac{x-\mu(\tau)}{1+s(\tau)}, \quad \tau=t, \quad \xi^{ \pm}(t)=\mu(\tau) \pm b(1+s(\tau)), \\
u(x, t)=f(z)+(s(\tau) z+\mu(\tau)) f^{\prime}(z)+w(z, \tau) .
\end{gathered}
$$

This transforms (5.3) into

$$
\begin{aligned}
w_{\tau}(z, \tau)= & \mathcal{L} w(z, \tau)+\mathcal{F}_{1}\left(z, w_{z z}(z, \tau), w_{z}(z, \tau), s(\tau), \mu(\tau)\right) \\
& +s^{\prime}(\tau) \mathcal{F}_{2}\left(z, z w_{z}(z, \tau), s(\tau), \mu(\tau)\right) \\
& +\mu^{\prime}(\tau) \mathcal{F}_{3}\left(z, w_{z}(z, \tau), s(\tau), \mu(\tau)\right),
\end{aligned}
$$

where $\mathcal{F}_{1}, \mathcal{F}_{2}$ and $\mathcal{F}_{3}$ contain also terms with $\mu$. The linear part is again $\mathcal{L} w=$ $w_{z z}-\frac{z}{2} w_{z}+\frac{1}{2} w$. The boundary conditions become

$$
w_{z}( \pm b, \tau)= \pm \frac{b}{2} w( \pm b, \tau)
$$

with $\mu$ and $s$ being given by

$$
\mu(\tau)=\frac{-w(b, \tau)+w(b, \tau)}{2} \text { and } s(\tau)=-\frac{-w(b, \tau)+w(b, \tau)}{2 b} .
$$

As before we obtain a fully nonlinear equation $w_{\tau}(z, \tau)=\mathcal{L} w(z, \tau)+\mathcal{F}$, where

$$
\mathcal{F}=\mathcal{F}\left(z, w_{z}, w_{z z}, w(b, \tau), w_{z}(b, \tau), w_{z z}(b, \tau), w(-b, \tau), w_{z}(-b, \tau), w_{z z}(-b, \tau)\right) .
$$

With $X=C^{0}([-b, b]), D(L)=\left\{\varphi \in C^{2}([-b, b]): \varphi^{\prime}( \pm b)= \pm \frac{b}{2} \varphi( \pm b)\right\}$, the realization $L$ of $\mathcal{L}$ is again sectorial, and its spectrum consists of the eigenvalues of

$$
\phi^{\prime \prime}-\frac{z}{2} \phi^{\prime}+\frac{1}{2} \phi=\lambda \phi, \quad \phi^{\prime}( \pm b)= \pm \frac{b}{2} \phi( \pm b) .
$$

Besides $\lambda_{1}=1$ with $\phi_{1}(z)=f^{\prime \prime}(z)$, we also have $\lambda_{2}=\frac{1}{2}$ with $\phi_{2}(z)=f^{\prime}(z)$. The other eigenvalues are again all negative. The unstable manifold consists of solutions focusing at another point or another time in the original $(x, t)$-variables. For the solutions in the stable manifold we obtain as before that

$$
\xi^{ \pm}(t)= \pm b(T-t)^{\frac{1}{2}}+O(T-t)^{\frac{1}{2}+\mu}
$$

for every $\mu<\lambda_{3}$.

\section{REFERENCES}

[1] S.B. Angenent, Analyticity of the interfaces of the porous media equation after the waiting time, Proc. A.M.S. 102, pp 329-336, 1988. MR 89f:35103

[2] M. Bertsch, D. Hilhorst \& C. Schmidt-Lainé, The well-posedness of a free boundary problem arising in combustion theory, Nonl. Anal. 23, pp 1211-1224, 1994. MR 95j:35241

[3] L.A. Caffarelli \& J.L. Vazquez, A free boundary problem for the heat equation arising in flame propagation, Trans. A.M.S. 347, pp 411-441, 1995. MR 95e:35097

[4] C.M. Brauner, J.Hulshof \& A. Lunardi, A general approach to stability in free boundary problems; in preparation.

[5] C.M. Brauner, A. Lunardi \& Cl. Schmidt-Lainé, Stability of travelling waves with interface conditions, Nonl. Anal. 19, pp 455-474, 1992. MR 93k:35145 
[6] C.M. Brauner, A. Lunardi \& Cl. Schmidt-Lainé, Multidimensional stability analysis of planar travelling waves, Appl. Math. Lett. 7(5), pp 1-4, 1994. CMP 96:01

[7] C.M. Brauner, A. Lunardi \& Cl. Schmidt-Lainé, Stability analysis in a multi-dimensional interface problem, Scuola Normale Superiore Pisa, Preprints di Matematica 3, 1996.

[8] C.M. Brauner, J.M. Roquejoffre \& Cl. Schmidt-Lainé, Stability of Travelling Waves in a Parabolic Equation with Discontinuous Source Term, Comm. Appl. Nonl. Anal. 2(4), pp 83-100, 1995. MR 96i:35052

[9] V.A. Galaktionov, J. Hulshof \& J.L. Vazquez, Extinction and focusing behaviour of spherical and annular flames described by a free boundary problem, J. Math. Pures et Appl., (76), pp 563-608, 1997. CMP 98:01

[10] D. Hilhorst \& J. Hulshof, An elliptic-parabolic problem in combustion theory: convergence to travelling waves, Nonl. Anal. 17, pp 519-546, 1991. MR 92g:35242

[11] D. Hilhorst \& J. Hulshof, A free boundary focusing problem, Proc. AMS 121, pp 1193-1202, 1994. MR 94j:35200

[12] J.D. Buckmaster \& G.S.S. Ludford, Theory of Laminar Flames, Cambridge University Press, 1982. MR 84f: 80011

[13] A. Lunardi, Analytic Semigroups and Optimal Regularity in Parabolic Problems, Birkhäuser 1995. MR 96e: 47039

Department of Mathematics, Leiden University, Niels Bohrweg 1, 2333 CA Leiden, The Netherlands

E-mail address: hulshof@wi.leidenuniv.nl

URL: http://www.wi.leidenuniv.nl/home/hulshof/

Mathématiques Appliquées de Bordeaux, Université Bordeaux I, 33405 Talence cedex, FRANCE

E-mail address: brauner@math.u-bordeaux.fr

CNRS UMR 128, Ecole Normale Supérieure de Lyon, 69364 Lyon Cedex 07, France

E-mail address: schmidt@umpa.ens-lyon.fr 